\title{
Examination of the predictive factors of the response to whole brain radiotherapy for brain metastases from lung cancer using MRI
}

\author{
SHURI AOKI $^{1,2}$, TOMONORI KANDA ${ }^{1}$, NORIYUKI MATSUTANI ${ }^{3}$, NOBUHIKO SEKI $^{4}$, \\ MASAFUMI KAWAMURA ${ }^{3}$, SHIGERU FURUI ${ }^{1}$ and HIDEOMI YAMASHITA ${ }^{2}$
}

\author{
${ }^{1}$ Department of Radiology, Teikyo University School of Medicine, Itabashi-ku, Tokyo 173-8605; ${ }^{2}$ Department of \\ Radiology, University of Tokyo Hospital, Bunkyo-ku, Tokyo 113-8655; Departments of ${ }^{3}$ General Thoracic Surgery \\ and ${ }^{4}$ Medical Oncology, Teikyo University School of Medicine, Itabashi-ku, Tokyo 173-8605, Japan
}

Received June 21, 2016; Accepted March 9, 2017

DOI: $10.3892 / 01.2017 .6264$

\begin{abstract}
Previous studies have been conducted on the prognostic factors for overall survival in patients with brain metastases (BMs) following whole brain radiotherapy (WBRT). However, there have been a small number of studies regarding the prognostic factors for the response of tumor to WBRT. The aim of the present study was to identify the predictive factors for the response to WBRT from the point of view of reduction of tumor using magnetic resonance imaging. A retrospective analysis of 62 patients with BMs from primary lung cancer treated with WBRT was undertaken. The effects of the following factors on the response to WBRT were evaluated: Age; sex; performance status; lactate dehydrogenase; pathology; existence of extracranial metastases; activity of extracranial disease; chemo-history; chest radiotherapy history; treatment term; $\gamma$-knife radiotherapy; diffusion weighted image signal intensity; tumor diameter; extent of edema and the edema/tumor (E/T) ratio. The association between the reduction of tumors and clinical factors was evaluated using logistic regression analysis. $\mathrm{P}<0.05$ was considered to indicate a statistically significant difference. The overall response ratio of this cohort was $54.8 \%$. In the univariate analysis, the response of tumors was associated with the presence of small cell lung carcinoma (SCLC; $\mathrm{P}=0.0007$ ), an $\mathrm{E} / \mathrm{T}$ ratio of $\geq 1.5(\mathrm{P}=0.048)$, and a median tumor diameter of $<20 \mathrm{~mm}(\mathrm{P}=0.014)$. In the multivariate analysis, the presence of SCLC $[\mathrm{P}=0.001$; odds ratio $(\mathrm{OR}), 17.152)$, an $\mathrm{E} / \mathrm{T}$ ratio of $\geq 1.5(\mathrm{P}=0.019 ; \mathrm{OR}, 9.526)$, and the presence of
\end{abstract}

Correspondence to: Dr Shuri Aoki, Department of Radiology, University of Tokyo Hospital, Tokyo, 3-7-1 Hongo, Bunkyo-ku, Tokyo 113-8655, Japan

E-mail: daisy.shuri@gmail.com

Key words: whole brain radiotherapy, predictive factor, brain metastases, response, lung cancer, magnetic resonance imaging, oligo-recurrence extracranial metastases $(\mathrm{P}=0.031 ; \mathrm{OR}, 4.875)$ were revealed to be independent predictive factors for the reduction of tumor. The following 3 factors were significantly associated with the response of tumors to WBRT: The presence of SCLC; an $\mathrm{E} / \mathrm{T}$ ratio of $\geq 1.5$; and the presence of extracranial metastases. The E/T ratio is a novel index that provides a simple and easy predictive method for use in a clinical setting.

\section{Introduction}

Brain metastases (BMs) are common in patients with cancer, with a rate of occurrence of $20-40 \%$ (1). The prognosis concerning the survival of patients with BMs is poor, with a 3-5-month median survival time, even when various types of treatments are attempted (2-4). Whole brain radiotherapy (WBRT) has been regarded as the standard treatment for BMs. One of the aims of WBRT has been to prevent the mortality of the patient from BMs, and to achieve longer overall survival (OS) (5); however, numerous studies have suggested that WBRT does not prolong the OS of patients with BMs (6-8). Advances in chemotherapy and molecular-targeted drugs have improved patient survival following the appearance of distant metastasis, including brain metastasis (9). For certain patients with brain metastases and good prognosis, stereotactic radiosurgery (SRS) and stereotactic radiotherapy (SRT) that have improved local control compared with WBRT have been recommended (10-18), and the primary objective of the WBRT has been increasingly to palliate neurological symptoms and to improve or maintain the patients' quality of life (QOL) (19) BMs cause headaches in $49 \%$ of patients, focal weakness in $30 \%$, mental disturbances in $32 \%$, gait ataxia in $21 \%$, seizures in $18 \%$ and other symptoms, leading to a lower QOL (8). Therefore, the control of BMs is necessary even in patients with poor survival times.

Regarding the prognostic factors for OS, A number of studies have been conducted in patients with BMs who received WBRT. The following parameters have been demonstrated to be prognostic factors: Karinofsky performance status (KPS); age; treatment history; extracranial metastases; systemic tumor activity; lactic dehydrogenase (LDH) level (20-27). 
Gaspar et al (1) suggested the recursive partitioning analysis (RPA) score as prognostic factors for clinical use, which includes following 3 factors; KPS; age; and the presence of extracranial metastases (1).

However, no predictive factors regarding the response to WBRT in reducing the size of BMs have been identified in previous studies. The aim of the present study was to identify predictive factors for the local control of WBRT.

\section{Materials and methods}

Ethical approval. The Teikyo University School of Medicine (Tokyo, Japan) ethics committee approved the present study. Written informed consent was waived due to the retrospective nature of the study. Patient information was anonymized prior to analysis.

Patient selection. A total of 94 patients with BMs from primary lung cancer were treated with WBRT using a palliative radiation dose (30-40 Gy) at Teikyo University School of Medicine between September 2010 and April 2013. The study was limited to patients who had undergone contrast-enhanced magnetic resonance imaging (MRI) within 1 month prior to WBRT, and brain imaging (contrast-enhanced computed tomography/MRI) using the same imaging modality prior and subsequent to WBRT. A total of 32 patients who had not undergone sufficient brain imaging were excluded. The remaining 62 patients (mean age, 67 years; age range, $50-85$ years) were evaluated.

Patient diagnoses. Patient outcomes were determined using medical records. The following parameters were evaluated: Performance status (PS); LDH level; pathology [small cell lung cancer (SCLC) vs. non-small cell lung cancer (NSCLC)]; extracranial metastases (yes vs. no); activity of extracranial disease (stable vs. progressive); chemo-history (yes vs. no); chest radiotherapy history (yes vs. no); treatment term [the interval between tumor diagnosis and WBRT (months)]; and use of $\gamma$-knife radiotherapy (yes vs. no).

The largest metastatic tumor was evaluated, excluding tumors with a history of previous $\gamma$-knife therapy. The following parameters were evaluated using patient imaging data prior and subsequent to WBRT: Tumor diameter; extent of edema, E/T ratio and diffusion weighted image (DWI) signal intensity.

The tumor diameter was the maximum diameter $(\mathrm{mm})$ detected as an enhanced lesion on the axial contrast enhanced T1-weighted images (WI). The extent of edema was expressed as the maximum length between the margin of the edema and that of the tumor on the axial T2-WI (Fig. 1). The extent of edema (E) was divided by the tumor diameter (T) and the association with RR was examined.

The DWI signal intensity was evaluated in the solid portion of the tumor in five stages as follows: 5 , higher compared with the cortex; 4 , iso to the cortex; 3 , higher compared with the white matter; 2 , iso to the white matter; and 1 , lower compared with the white matter (Fig. 2). Image analysis was conducted by two radiology specialists by consensus (T.K. and S.A., with 11 and 5 years of experience, respectively), who were blinded to the clinical data. In clinical practice, the treatment effect is
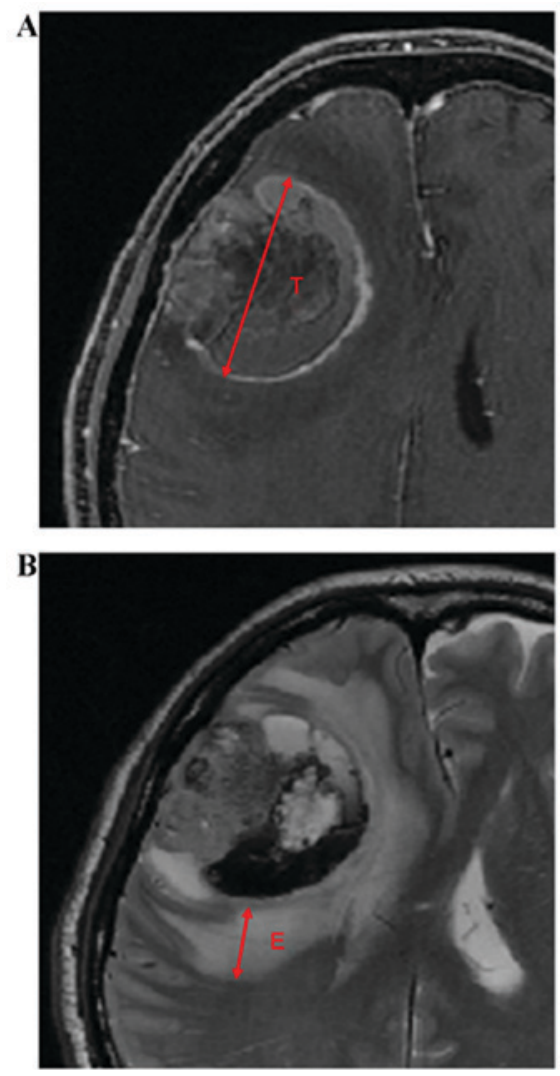

Figure 1. Definition of the E/T ratio. (A) The tumor diameter was measured as the maximum length of tumor on a contrast enhanced T1-weighted image. (B) The extent of edema was measured as the maximum length between the margin of the edema and that of the tumor on T2-weighted image. The E/T ratio was defined as the $\mathrm{E} / \mathrm{T}$ ratio. $\mathrm{E} / \mathrm{T}$, edema/tumor.

evaluated in accordance with Response Evaluation Criteria in Solid Tumors version 4.0 (RECIST v4.0), short-term efficacy was classified into 4 groups: complete response (CR); partial response (PR); stable disease (SD); and progressive disease (PD). In the current study, the patients were divided into two groups, response group $(\mathrm{CR}+\mathrm{PR})$ and non-response group $(\mathrm{SD}+\mathrm{PD})$.

Statistical analysis. Univariate and multivariate analyses were performed to identify significant prognostic factors. Logistic regression analysis with backward elimination was used to evaluate the association between treatment effectiveness and the following factors: Age; sex; PS; LDH; pathology; extracranial metastases; extent of extracranial disease; chemo-history; chest radiotherapy history; treatment term; use of $\gamma$-knife radiotherapy; DWI signal intensity; tumor diameter; extent of edema; and the E/T ratio. All statistical analyses were performed using software (PASW Statistics, v.21.0: IBM SPSS, Armonk, NY, USA), $\mathrm{P}<0.05$ was considered to indicate a statistically significant difference.

\section{Results}

The patient characteristics are summarized in Table I. A total of 35 (57\%) patients exhibited a PS $\geq 2,20$ (32\%) exhibited SCLC, $35(57 \%)$ exhibited extracranial metastases, 37 (60\%) suffered from progressive extracranial disease, $37(60 \%)$ demonstrated a chemo-history, 7 (11\%) exhibited a history of chest radiotherapy, 
Table I. Patient characteristics.

\begin{tabular}{lcr}
\hline Parameters & No. (range) & $\%$ \\
\hline Age, years & 67 & \\
Sex & & \\
Male & 46 & 74.2 \\
Female & 16 & 25.8 \\
Performance status & & \\
0 & 4 & 6.5 \\
1 & 23 & 37.1 \\
2 & 12 & 19.4 \\
3 & 17 & 27.4 \\
4 & 6 & 9.7 \\
Histological type & & \\
NSCLC & 42 & 67.7 \\
Adenocarcinoma & 34 & 54.8 \\
Squamous cell carcinoma & 2 & 3.2 \\
Others & 6 & 9.7 \\
SCLC & 20 & 32.3
\end{tabular}

Extracranial metastases

Yes

No

Activity of extracranial tumor

Stable

Progressive

Treatment term from diagnosis ${ }^{\mathrm{a}}$

Chemotherapy regimen

$$
2
$$$$
0
$$$$
1
$$$$
3
$$$$
4
$$

Chest radiotherapy history
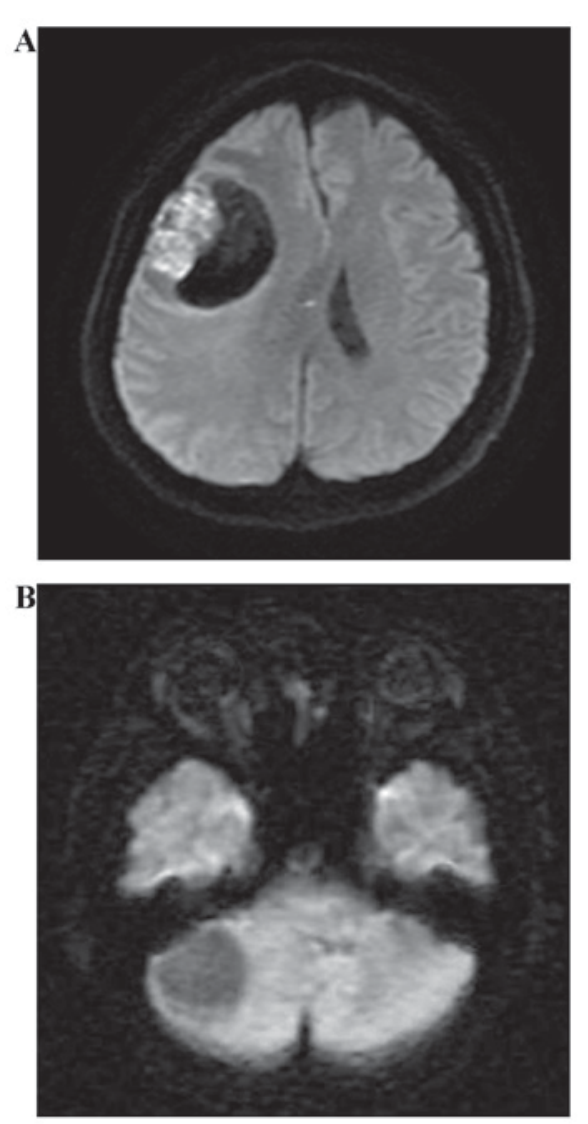

Figure 2. Evaluation of DWI signal intensity. The DWI signal intensity was evaluated in the solid portion of the tumor in five stages as follows: 5, higher compared with the cortex; 4 , iso to the cortex; 3 , higher compared with the white matter; 2 , iso to the white matter; and 1, lower compared with the white matter. (A) This lesion existed in the high signal intensity solid portion and low signal cystic portion on DWI, and the signal intensity was classified as grade 5. (B) This lesion was solid on the contrast enhanced T1-weighted image, and demonstrated lower signal intensity compared with the white matter on DWI; the signal intensity was classified as grade 1. DWI, diffusion weighted image.

$$
\text { Yes }
$$$$
\text { No }
$$

Tumor diameter, $\mathrm{mm}^{\mathrm{a}}$

Edema diameter, $\mathrm{mm}^{\mathrm{a}}$

Edema/tumor ratio

\section{DWI Intensity}

$$
\text { 1-4 }
$$$$
5
$$

Response

$$
\mathrm{CR}+\mathrm{PR}
$$

SD+PD

\section{3}

88.7

$$
58.1
$$

a'Data presented as the median. NSCLC, non-small cell lung cancer; SCLC, small cell lung cancer; DWI, diffusion weighted image; $\mathrm{CR}+\mathrm{PR}$, complete response+partial response; $\mathrm{SD}+\mathrm{PD}$, stable disease+progressive disease. and $9(15 \%)$ demonstrated history of $\gamma$-knife radiotherapy. An $\mathrm{E} / \mathrm{T}$ ratio $\geq 1.5$ was revealed in $11(18 \%)$ patients.

The overall response ratio (RR) of the cohort was $54.8 \%$. In the univariate analyses, the response of tumors to WBRT was associated with the presence of small cell lung carcinoma (SCLC; $\mathrm{P}=0.0007), \mathrm{E} / \mathrm{T}$ ratio $\geq 1.5(\mathrm{P}=0.048)$, and median tumor diameter of $<20 \mathrm{~mm}(\mathrm{P}=0.014)$. The result of univariate analysis is described in Table II. In the multivariate analysis, the following 3 factors, presence of SCLC $[\mathrm{P}=0.001$; odds ratio (OR), 17.152], an $\mathrm{E} / \mathrm{T}$ ratio $\geq 1.5$ ( $\mathrm{P}=0.019$; $\mathrm{OR}$, 9.526); and presence of extracranial metastases $(\mathrm{P}=0.031$; OR, 4.875) were revealed to be independent prognostic parameters for treatment effects (Table III).

\section{Discussion}

Data from 62 lung cancer patients with BMs who underwent WBRT were retrospectively reviewed; the overall RR was $54.8 \%$, which was similar to previous studies $(4,28)$. In WBRT for BMs from lung cancer, BMs with the following characteristics are expected to have a higher RR: From SCLC compared with NSCLC (OR, 17.152); an E/T ratio of $\geq 1.5$ rather opposed 
Table II. Result of univariate analysis.

\begin{tabular}{|c|c|c|c|}
\hline Parameters & Mean & Standard deviation & P-value \\
\hline Sex & & & 0.658 \\
\hline Male & 0.5 & 0.516 & \\
\hline Female & 0.565 & 0.501 & \\
\hline Performance status & & & 0.922 \\
\hline $0-1$ & 0.556 & 0.506 & \\
\hline $2-4$ & 0.543 & 0.505 & \\
\hline Symptom & & & 0.217 \\
\hline Yes & 0.625 & 0.492 & \\
\hline No & 0.467 & 0.507 & \\
\hline Histological type & & & 0.0007 \\
\hline Non-small cell lung cancer & 0.405 & 0.5 & \\
\hline Small cell lung cancer & 0.85 & 0.366 & \\
\hline Extracranial metastases & & & 0.882 \\
\hline Yes & 0.541 & 0.505 & \\
\hline No & 0.56 & 0.507 & \\
\hline Activity of extracranial tumor & & & 0.154 \\
\hline Stable & 0.444 & 0.506 & \\
\hline Progressive & 0.629 & 0.49 & \\
\hline Treatment term from diagnosis & & & 0.361 \\
\hline$\leq 10$ & 0.6 & 0.497 & \\
\hline$>10$ & 0.481 & 0.509 & \\
\hline Chest radiotherapy history & & & 0.899 \\
\hline Yes & 0.545 & 0.503 & \\
\hline No & 0.571 & 0.535 & \\
\hline$\gamma$-knife & & & 0.166 \\
\hline Yes & 0.585 & 0.497 & \\
\hline No & 0.333 & 0.5 & \\
\hline Chemotherapy history & & & 0.841 \\
\hline With & 0.538 & 0.505 & \\
\hline Without & 0.565 & 0.507 & \\
\hline Lactate dehydrogenase & & & 0.402 \\
\hline$<250$ & 0.59 & 0.498 & \\
\hline$\geq 250$ & 0.478 & 0.511 & \\
\hline Diffusion weighted image intensity & & & 0.25 \\
\hline $1-4$ & 0.462 & 0.508 & \\
\hline 5 & 0.611 & 0.494 & \\
\hline Tumor diameter, mm & & & 0.0144 \\
\hline$\geq 20$ & 0.659 & 0.48 & \\
\hline$<20$ & 0.333 & 0.483 & \\
\hline Edema diameter, mm & & & 0.361 \\
\hline$\leq 10$ & 0.6 & 0.497 & \\
\hline$>10$ & 0.481 & 0.509 & \\
\hline Edema/tumor ratio & & & 0.0484 \\
\hline$\leq 1.5$ & 0.49 & 0.504 & \\
\hline$>1.5$ & 0.818 & 0.404 & \\
\hline
\end{tabular}

to $<1.5(\mathrm{OR}, 9.526)$; and an absence rather than a presence of extracranial metastases (OR, 4.875).
Patients with SCLC exhibited an improved RR compared with patients with NSCLC. It is widely known that SCLC 
Table III. Results of logistic regression analysis.

$95 \%$ confidence interval

\begin{tabular}{lcccc}
\cline { 2 - 4 } Parameter & Odds ratio & Lower & Upper & P-value \\
\hline Pathology & 17.152 & 3.242 & 90.758 & 0.001 \\
Extracranial metastases & 4.875 & 1.156 & 20.549 & 0.031 \\
Edema/tumor ratio $>1.5$ & 9.526 & 1.453 & 62.459 & 0.019 \\
\hline
\end{tabular}

Table IV. Previous studies examining predictive factors for the patients with BMs who underwent WBRT.

Author (publication year)

Predicting factors for vital prognosis

(Refs.)

Gaspar et al, 1997

Lagerwaard et al, 1999

Partl et al, 2016

Windsor et al, 2013

Rades et al, 2013

Zimm et al, 1981

Sperduto et al, 2008

Komatsu et al, 2013

Mayahara et al, 2012

Zhu et al, 2014
KPS, age, extracranial metastases

PS, response to steroid treatment, systemic tumor activity,

LDH site of primary tumor, age, number of BM, sex

$\mathrm{KPS} / \mathrm{LDH}$ index

Age, sex, primary cancer, time to WBRT from the primary cancer diagnosis

Sex, KPS, extracranial metastases

PS, age, symptom of headache/visual disturbance/impaired consciousness

KPS, age, extracranial metastases, number of BMs

Histological type, EGFR mutation (use of EGFR-TKI)

KPS, sex, activity of extracranial disease, time to develop BM,

use of chemotherapy following WBRT

Plasma fibrinogen

(1)

PS, performance status; KPS, Karnofsky performance status; LDH, lactate dehydrogenase; BM, brain metastases; EGFR, epidermal growth factor receptor; TKI, tyrosine kinase inhibitor; WBRT, whole brain radiation therapy.

exhibits high radiosensitivity (29-31). In addition, it has been suggested that BMs also demonstrate high radiosensitivity, similar to that observed in the primary tumor (32). The data of the present study agree with those from previous studies.

In the current study, BMs with an $\mathrm{E} / \mathrm{T}$ ratio of $\geq 1.5$ are expected to exhibit a higher RR rather than BMs with an $\mathrm{E} / \mathrm{T}$ ratio of $<1.5$. Peritumoral edema is caused by disturbed vascular permeability that enables an indiscriminate escape of plasma proteins from the blood into BMs or the peritumoral regions of the brain (33). The present study was not able to explain the immediate reason why BMs with relatively extensive edema $(\mathrm{E} / \mathrm{T}$ ratio $\geq 1.5)$ exhibited an improved response to WBRT. However, E/T ratios may provide useful information for predicting therapeutic efficacy.

DWI produces a signal intensity that reflects the cell density of a tumor and correlates with its histopathological image; therefore, it is believed to be useful in predicting the treatment effectiveness for esophageal, pharyngeal and cervical cancer (34-37). DWI is also considered to be useful in predicting the efficacy of g-knife therapy for BMs (38). However, no statistically significant difference in RR has been observed for BMs derived from lung cancer with different DWI signal intensities; these BMs are occasionally accompanied by hemorrhage, necrosis and mucous production that present with various signal intensities (39). Therefore, it has been concluded that DWI would not predict the therapeutic effect for BMs from lung cancer.
The presence of extracranial metastases also correlated with an improved RR. Notably, it is a rather reverse result to the studies concerning OS $(17,24)$. The RR results are explained as follows, low differentiated tumors often cause distant metastasis and exhibit high radiosensitivity, and this factor may have affected the results of the present study.

There are numerous studies that have identified prognostic factors focused on OS in patients with BM who underwent WBRT. For example, the RPA score proposed by Gaspar et al (1), which is widely used in clinical settings, includes KPS, age and the presence of an active extracranial lesion. Other factors including sex, symptom and response to steroid treatment have been indicated as predictive factors (Table IV). These factors are not equal with the result of the present study focused on local control.

The majority of patients who receive WBRT demonstrate poor health; $51 \%$ of these patients exhibit an RPA class 3 score, which is equivalent to a KPS $<70$ (19). Thus, a number of the factors that are useful for prognosis of WBRT regarding OS reflect the general condition of the patients and their general disease status, rather than reflecting the response to WBRT. This may be one reason why the factors useful for predicting vital prognosis do not necessarily coincide with the response to WBRT. Therefore, it is considered that the vital prognosis of patients and the response of tumors to WBRT should have separate predictive factors, and it would be useful to employ 
these in combination, to make decisions concerning the treatment of the patients.

In cases with poor response to WBRT expected, the course of treatment for each patient should potentially be reconsidered, according to their vital prognosis. That is, for patients with predicted poor responses to WBRT and poor vital prognosis, palliative care without WBRT should be considered to improve QOL (40). For patients with expected poor response to WBRT but good vital prognosis, additional radical treatment for BMs should be considered, such as SRS or SRT $(3,4,10)$.

Recently, the novel hypothesis of oligometastases has been proposed, define as patients with a limited number of metastases (oligometastases), may benefit of survival from curative local therapy for metastases $(11-13)$. In addition, Niibe et al $(12,14)$ defined oligo-recurrence and this is similar to oligometastases, but the primary lesion is also controlled $(12,14)$.

A number of studies are have investigated the use of curative radiotherapy for oligometastases or oligo-reccurence and particularly in oligo-recurrence, it was demonstrated to be a favorable factor for OS and relapse-free survival, and it was also associated with improved local control of the metastases $(12,15,16)$. This is also true for SRS/SRT for BMs $(17,18)$.

In the present study about WBRT, almost all patients exhibited uncontrolled primary lesions, multi-organ metastases or numerous BMs. Thus, it was not possible to neither examine enough oligo-recurrence cases nor indicate the immediate association between the response of BMs and the state of oligo-recurrence. However, further clinical studies examining the association between the local control of patients with BMs undergoing WBRT and the state of oligo-recurrence, may lead to the identification of an important predictive factor for appropriate treatment decisions.

The present study had several limitations. The number of patients studied was 64 , which is a small sample size. In addition, the study was retrospective. The majority of the data used were extracted from previous medical records, which made it difficult to evaluate the association between the improvement of the symptom of BMs and the response of tumor to WBRT. It was not possible to examine the EGFR mutation status of the patients, or other genomic information. Regarding the evaluation of the DWI signal intensity, an evaluation using an apparent diffusion coefficient (ADC) map was not performed. The use of an ADC map is considered preferable for more effective evaluation of the nature of tumors. However, DWI signal intensity, which is clinically convenient, was used in the present study. It will be necessary to validate these data by studying more patients, or by performing a prospective study, prior to their application in an actual clinical setting.

In conclusion, predictive factors for the response of BMs to WBRT for patients with lung cancer were investigated. The following three factors were significantly associated to the response to WBRT: The presence of SCLC, an E/T ratio $\geq 1.5$ and the presence of extracranial metastases. The identification of predictive factors concerning the response to WBRT may provide useful information to facilitate the selection of adequate individual treatments for patients with lung cancer with BM prior to WBRT.

\section{References}

1. Gaspar L, Scott C, Rotman M, Asbell S, Phillips T, Wasserman T, McKenna WG and Byhardt R: Recursive partitioning analysis (RPA) of prognostic factors in three Radiation therapy oncology group (RTOG) brain metastases trials. Int J Radiat Oncol Biol Phys 37: 745-751, 1997.

2. Lagerwaard FJ, Levendag PC, Nowak PJ, Eijkenboom WM, Hanssens PE and Schmitz PI: Identification of prognostic factors in patients with brain metastases: A review of 1292 patients. Int J Radiat Oncol Biol Phys 43: 795-803, 1999.

3. Gaspar LE, Mehta MP, Patchell RA, Burri SH, Robinson PD, Morris RE, Ammirati M, Andrews DW, Asher AL, Cobbs CS, et al: The role of whole brain radiation therapy in the management of newly diagnosed brain metastases: A systematic review and evidence-based clinical practice guideline. J Neurooncol 96: 17-32, 2010.

4. Postmus PE, Haaxma-Reiche H, Gregor A, Groen HJ, Lewinski T and Scolard T: Brain-only metastases of small cell lung cancer; efficacy of whole brain radiotherapy. An EORTC phase II study. Radiother Oncol 46: 29-32, 1998.

5. Gao Y, Gao F, Ma JL and Zhao DL: Palliative whole-brain radiotherapy and health-related quality of life for patients with brain metastasis in cancer. Neuropsychiatr Dis Treat 11: 2185-2190, 2015.

6. Chao JH, Phillips R and Nickson JJ: Roentgen-ray therapy of cerebral metastases. Cancer 7: 682-689, 1954.

7. Order SE, Hellman S, Von Essen CF and Kligerman MM: Improvement in quality of survival following whole-brain irradiation for brain metastases. Radiology 91: 149-153, 1968.

8. Tsao MN, Lloyd NS, Wong RK, Rakovitch E, Chow E and Laperriere N; Supportive Care Guidelines Group of Cancer Care Ontario's Program in Evidence-based Care: Radiotherapeutic management of brain metastases: A systematic review and meta-analysis. Cancer Treat Rev 31: 256-273, 2005.

9. Zimmermann S, Dziadziuszko R and Peters S: Indications and limitations of chemotherapy and targeted agents in non-small cell lung cancer brain metastases. Cancer Treat Rev 40: 716-722, 2014.

10. Niibe Y, Karasawa K, Nakamura O, Shinoura N, Okamoto K, Yamada R, Fukino K and Tanaka Y: Survival benefit of stereotactic radiosurgery for metastatic brain tumors in patients with controlled primary lesions and no other distant metastases. Anticancer Res 23: 4157-4159, 2003.

11. Hellman S and Weichselbaum RR: Oligometastases. J Clin Oncol 13: 8-10, 1995.

12. Niibe Y and Hayakawa K: Oligometastases and oligo-recurrence: The new era of cancer therapy. Jpn J Clin Oncol 40: 107-111, 2010.

13. Niibe Y and Chang JY: Novel insights of oligometastases and oligo-recurrence and review of the literature. Pulm Med 2012: 261096, 2012.

14. Niibe Y, Chang JY, Onishi H, Salama J, Hiraki T and Yamashita H: Oligometastases/Oligo-recurrence of lung cancer. Pulm Med 2013: 438236, 2013.

15. Yamashita H, Niibe Y, Yamamoto T, Katsui K, Jingu K, Kanazawa S, Terahara A and Nakagawa K: Lung stereotactic radiotherapy for oligometastases: Comparison of oligo-recurrence and sync oligometastases. Jpn J Clin Oncol 46: 687-791, 2016.

16. Won YK, Lee JY, Kang YN, Jang JS, Kang JH, Jung SL, Sung SY, Jo IY, Park HH, Lee DS, et al: Stereotactic radiosurgery for brain metastasis in non-small cell lung cancer. Radiat Oncol J 33: 207-216, 2015.

17. Wang TJ, Saad S, Qureshi YH, Jani A, Isaacson SR, Sisti MB, Bruce JN, McKhann GM II, Lesser J, Cheng SK, et al: Outcomes of gamma knife radiosurgery, bi-modality \& tri-modality treatment regimens for patients with one or multiple brain metastases: The Columbia University Medical Center experience. J Neurooncol 122: 399-408, 2015.

18. Niibe Y, Nishimura T, Inoue T, Karasawa K, Y, Jingu K and Shirato H: Oligo-recurrence predicts favorable prognosis of brain-only oligometastases in patients with non-small cell lung cancer treated with stereotactic radiosurgery or stereotactic radiotherapy: A multi-institutional study of 61 subjects. BMC Cancer 16: 659, 2016.

19. Mulvenna PM: The management of brain metastases in patients with non-small cell lung cancer-is it time to go back to the drawing board? Clin Oncol (R Coll Radiol) 22: 365-373, 2010. 
20. Partl R, Fastner G, Kaiser J, Kronhuber E, Cetin-Strohmer K, Steffal C, Böhmer-Breitfelder B, Mayer J, Avian A and Berghold A: KPS/LDH index: A simple tool for identifying patients with metastatic melanoma who are unlikely to benefit from palliative whole brain radiotherapy. Support Care Cancer 24: 523-528, 2016

21. Windsor AA, Koh ES, Allen S, Gabriel GS, Yeo AE, Allison R, van der Linden YM and Barton MB: Poor outcomes after whole brain radiotherapy in patients with brain metastases: Results from an international multicentre cohort study. Clin Oncol (R Coll Radiol) 25: 674-680, 2013.

22. Rades D, Dziggel L, Nagy V, Segedin B, Lohynska R, Veninga T, Khoa MT, Trang NT and Schild SE: A new survival score for patients with brain metastases who received whole-brain radiotherapy (WBRT) alone. Radiother Oncol 108: 123-127, 2013.

23. Zimm S, Wampler GL, Stablein D, Hazra T and Young HF: Intracerebral metastases in solid-tumor patients: Natural history and results of treatment. Cancer 48: 384-394, 1981.

24. Sperduto PW, Berkey B, Gaspar LE, Mehta M and Curran W: A new prognostic index and comparison to three other indices for patients with brain metastases: An analysis of 1,960 patients in the RTOG database. Int J Radiat Oncol Biol Phys 70: 510-514, 2008.

25. Komatsu T, Kunieda E, Oizumi Y, Tamai Y and Akiba T: Clinical characteristics of brain metastases from lung cancer according to histological type: Pretreatment evaluation and survival following whole-brain radiotherapy. Mol Clin Oncol 1: 692-698, 2013.

26. Mayahara H, Sumi M, Ito Y, Sekii S, Takahashi K, Inaba K, Kuroda Y, Murakami N, Morota M and Itami J: Effect of chemotherapy on survival after whole brain radiation therapy for brain metastases: A single-center retrospective analysis. J Cancer Res Clin Oncol 138: 1239-1247, 2012.

27. Zhu JF, Cai L, Zhang XW, Wen YS, Su XD, Rong TH and Zhang LJ: High plasma fibrinogen concentration and platelet count unfavorably impact survival in non-small cell lung cancer patients with brain metastases. Chin J Cancer 33: 96-104, 2014.

28. Barnes EA, Chow E, Tsao MN, Bradley NM, Doyle M, Li K, Lam K and Danjoux C: Physician expectations of treatment outcomes for patients with brain metastases referred for whole brain radiotherapy. Int J Radiat Oncol Biol Phys 76: 187-192, 2010.

29. Kuremsky JG, Urbanic JJ, Petty WJ, Lovato JF, Bourland JD, Tatter SB, Ellis TL, McMullen KP, Shaw EG and Chan MD Tumor histology predicts patterns of failure and survival in patients with brain metastases from lung cancer treated with gamma knife radiosurgery. Neurosurgery 73: 641-647, 2013.
30. Seute T, Leffers P, Wilmink JT, ten Velde GP and Twijnstra A: Response of asymptomatic brain metastases from small-cell lung cancer to systemic first-line chemotherapy. J Clin Oncol 24: 2079-2083, 2006.

31. Bohlen G, Meyners T, Kieckebusch S, Lohynska R, Veninga T, Stalpers LJ, Schild SE and Rades D: Short-course whole-brain radiotherapy (WBRT) for brain metastases due to small-cell lung cancer (SCLC). Clin Neurol Neurosurg 112: 183-187, 2010.

32. Schuette W: Treatment of brain metastases from lung cancer: Chemotherapy. Lung Cancer 45 (Suppl 2): S253-S257, 2004.

33. Zhang $\mathrm{M}$ and Olsson $\mathrm{Y}$ : Hematogenous metastases of the human brain-characteristics of peritumoral brain changes: A review. J Neurooncol 35: 81-89, 1997.

34. Wang L, Han C, Zhu S, Shi G, Wang Q, Tian H, Kong J and Zhang A: Investigation of using diffusion-weighted magnetic resonance imaging to evaluate the therapeutic effect of esophageal carcinoma treatment. Oncol Res Treat 37: 112-116, 2014.

35. Chen Y, Liu X, Zheng D, Xu L, Hong L, Xu Y and Pan J: Diffusion-weighted magnetic resonance imaging for early response assessment of chemoradiotherapy in patients with nasopharyngeal carcinoma. Magn Reson Imaging 32: 630-637, 2014.

36. Hong J, Yao Y, Zhang Y, Tang T, Zhang H, Bao D, Chen Y and Pan J: Value of magnetic resonance diffusion-weighted imaging for the prediction of radiosensitivity in nasopharyngeal carcinoma. Otolaryngol Head Neck Surg 149: 707-713, 2013.

37. Mardor Y, Roth Y, Ochershvilli A, Spiegelmann R, Tichler T, Daniels D, Maier SE, Nissim O, Ram Z, Baram J, et al: Pretreatment prediction of brain tumors' response to radiation therapy using high b-value diffusion-weighted MRI. Neoplasia 6: 136-142, 2004.

38. Lee CC, Wintermark M, Xu Z, Yen CP, Schlesinger D and Sheehan JP: Application of diffusion-weighted magnetic resonance imaging to predict the intracranial metastatic tumor response to gamma knife radiosurgery. J Neurooncol 118: 351-361, 2014.

39. Osborn AG: Osborn's Brain: Imaging, Pathology and Anatomy. Lippincott Williams \& Wilkins, Philadelphia, PA, 2012.

40. Tsao MN, Rades D, Wirth A, Lo SS, Danielson BL, Gaspar LE, Sperduto PW, Vogelbaum MA, Radawski JD, Wang JZ, et al: Radiotherapeutic and surgical management for newly diagnosed brain metastasis(es): An American Society for Radiation Oncology evidence-based guideline. Pract Radiat Oncol 2: 210-225, 2012. 